\title{
NUMERICAL ANALYSIS OF ANTI VORTEX DEVICES PERFORMANCE TO SUPPRESS THE AIR ENTRAINING VORTEX IN PUMP SUMP
}

\author{
RAHUL PALIWAL $^{1 *} \&$ ANANT JHAVERI ${ }^{2}$ \\ ${ }^{I}$ Assistant Professor, Department of Chemical Engineering, NMIMS Mukesh Patel School of Technology \\ Management \& Engineering, Mumbai, India \\ ${ }^{2}$ Associate Professor, Department of Chemical Engineering, NMIMS Mukesh Patel School of Technology
}

Management \& Engineering, Mumbai, India

\begin{abstract}
Non-uniform swirling flow and air entraining vortex are among the important causes of reduction in efficiency of hydraulic machines such as pumps, turbines etc. A numerical simulation based study presented in this paper is to evaluate the effect of Anti Vortex Devices (AVD) in minimizing the effect of air entraining surface vortex in the intake section of centrifugal pump. In proposed methodology, Fast Fourier Transform (FFT) analysis is performed on the pressure fluctuation data, obtained from Large Eddy Simulation (LES) model. Numerical results are obtained for a rectangular sump with and without AVD. FFT analysis shows the presence of large and small eddies in the sump. Effectiveness of $A V D$, in breaking down the large eddies to prevent the formation of vortex, can be clearly observed in FFT results. Triangular and modified AVD performed effectively in reducing the air entraining surface vortex.

KEYWORDS: Large Eddy Simulation, Fast Fourier Transform, Vortex, Anti-Vortex Devices \& Hydraulic Machines
\end{abstract}

Received: Feb 12, 2020; Accepted: Mar 02, 2020; Published: Mar 13, 2020; Paper Id.: IJMPERDAPR202051

\section{INTRODUCTION}

As an answer to the global climatic challenge, the world's energy needs should be fulfilled via sustainable pathways. Hence, there is an increase in the research related to optimization of energy utilization of the existing methods or the development of new green technologies. Sustainable utilization of energy increased the demand for assessment and enhancement of the efficiency of different processes. Large capacity pumping is an integral part of different hydropower project, nuclear power projects, desalination, irrigation etc. that consume lots of energy. Bouzidi [1] presented new sizing methods for photo voltaic (PV) water pumping system and observed that the wrong storage tank design affect the efficiency of the system along with the incorrect array of PV module. Energy flow analysis in agriculture have been done by Bartzas et al. [2], they pointed out that the machinery used in irrigation and different operations is the most critical input. According to the report of the European Commission, nearly 22 percent of energy supplied by electric motors around the world is used to power up pumping systems.[3]

American National Standard Institution (ANSI) [4], the British Standard Institution (BSI)[5] and the Japan Society of Mechanical Engineers (JSME) [6] issued guidelines for designing of pump sump. Still, there is reduction in pump efficiency due to inappropriate sump design, water level or outflow cause swirl flow or vortices, which is the main cause of reduction in pump efficiency. If it is not solved, then it will reduce the pump effectiveness and may increase the operation cost [7]. According to Turbo machinery Society (TSJ) of Japan standard S2002:2005 [8], generally, two types of vortices can be found in the intake section of the turbines and pump sump. Air entraining 
free surface vortex and submerged vortex. Kumar et al. [9] elaborately discussed the cavitations related to the hydro turbine and gave the detailed analysis of the causes and effects of cavitation on the efficiency of the turbine. Zhang et al. [10] elaborately discussed the types of vortices and also presented different experimental techniques to visualize the vortex structure. Ahn et al. [11] studied the effect of the surface vortices on the intake flow for the tidal power station and concluded that surface vortices affect the net head and flow rate, which will be enhanced with a decrease in water level. Goyal et al. [12] gave a comprehensive review of the model Francis turbine for PIV measurements. He concluded that unsteady and flow with high vorticity may cause a dip in the efficiency of the turbine.

Rajendran et al. [13] observed the flow behavior in a rectangular sump for flow through vertical pipe with particle image velocimetry (PIV) and performed numerical analysis. Rho et al. [14] studied the flow in sump for pump station numerically based on Computational Fluid Dynamics (CFD). The Turbo machinery Society of Japan (TSJ) [8] suggested some changes in the standard of pump sump and studied the applicability of numerical models to the same. Okamura et al. [15] performed experiments based on TSJ test and reproduced the results with numerical models. In this paper, results reported by Okamura et al. [15] are taken as benchmark case to validate the LES model and to assess the performances of Anti Vortex Devices (AVD) for reducing the vortices in the system.

ANSI [4] recommended the use of AVD for reducing the vorticity in the flow, but lack of clarity on dimension forced researchers to develop a variety of AVDs. Generally, two different types of AVDs, viz. co-axial with intake pipe and floor splitter, are found in the literature. Co- axial AVD kept at the floor of the sump in axial plane with intake pipe. Floor splitter AVDs are usually used to divide the flow in two half. Choi et al. [16] numerically simulated the pumping station with multiple pumps and studied the effect of varying on vortex formation in a sump with multiple pumps. Kang et al. [17] performed experiments with rectangular and trapezoidal cross section floor splitter to reduce the vorticity in the sump. Based on the values of swirl angle the trapezoidal AVD effectively reduce the vorticity. Floor cone type AVD with serrated surface modification can effectively minimize the formation of submerged vortex [18]. Norizan et al. [19] studied the effect of floor splitter AVD in pump sump to increase the pump efficiency. They modified the trapezoidal AVD suggested by [17] into triangular and rounded top cross section. Kim et al. [20] found that the performance of AVD will be more effective with increased flow rates.

In the literature evaluation of AVDs, performance had been done based on the values of swirl angle and vorticity value. But these results could not predict the performance of AVD against surface vortices. Moreover, there is a need for comparative analysis of the performance of the different AVDs for the same operating conditions. In this paper, Fast Fourier Transform (FFT) based method has been utilized for the assessment of the performance of the AVDs for minimizing the vortex causing conditions. Characteristic frequency can be assigned for a particular AVD on the basis of power spectrum obtained from FFT analysis.

\section{METHODOLOGY}

\subsection{Simulation Model Description}

Flow simulation for a rectangular reservoir model was conducted to investigate the effectiveness of the AVDs for minimizing the vortex effect. Simulation did not include pump guide vanes. The geometry in the present study was based on the benchmark study by Okamura et al. [15] on a scale model. While using scale model for conducting the hydraulic test, we need to maintain certain conditions for Reynold's and Weber number. Padmanabhan and Hecker [21] showed that the occurrence of the vortex has no significant scale effect, if Reynold's number is greater than $3 \times 10^{4}$. Jain et al [22] reported no effect of surface tension on the critical submergence if Weber number is greater than 120. Figure 1 represents the model geometry and 
all the dimensions are taken in terms of pipe diameter D. In this case D taken as $0.145 \mathrm{~m}$ as taken in the experimental study of Okamura et al [15] and outer diameter D1 as $0.150 \mathrm{~m}$. The length of the rectangular channel taken as 4.5D and pipe was situated at a distance $\mathrm{L} 1=0.76 \mathrm{D}$. The distance from the side wall $\mathrm{W} 1$ and $\mathrm{W} 2$ are taken as $0.96 \mathrm{D}$ and $1.1 \mathrm{D}$ respectively. The Height of water $\mathrm{H}$ kept constant at 2D and pipe inlet is kept with a clearance of 0.69D from floor.
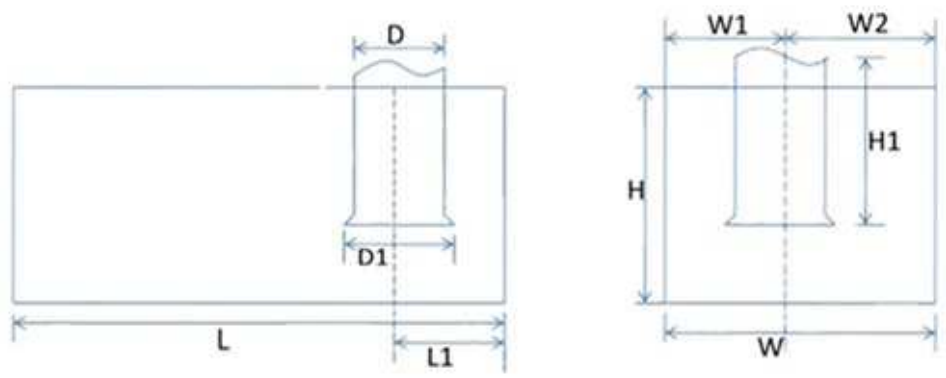

Figure 1: Geometrical Details of the Simulation Model.

In the present study, the basic design of the AVDs were taken from the literature. The height of all the AVDs, AVD-H, was kept constant as 0.1D. AVD-L for axis symmetry AVD's taken as 1D and for floor splitter AVD's AVD-L' was taken as 1.27D. Height of side wall fillet AVD-e was taken as 0.24D. And angle for cutting plane $\alpha$ was $45^{\circ}$. Figure 2 shows the location of Anti vortex device.

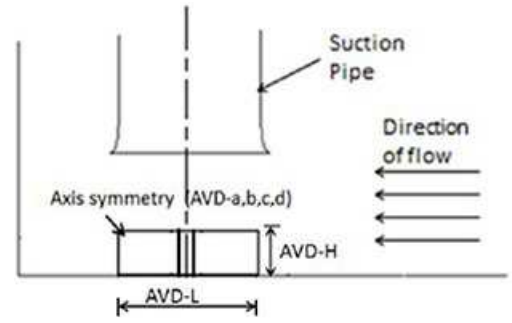

(a)

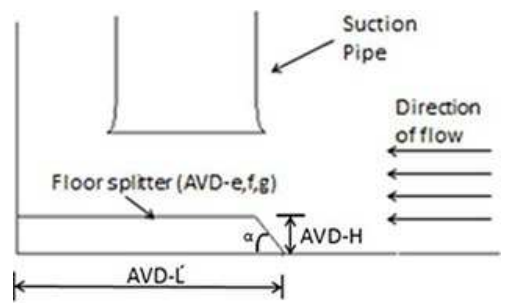

(b)

Figure 2: Location of Anti Vortex Devices. (a) Axis Symmetry. (b) Floor Splitter.

Figure 3 shows the shapes of Anti Vortex devices used in the study as vortex mitigation device. In the simulation as a comparative analysis, AVDs were taken from the literature with modified dimension to keep the similarity.

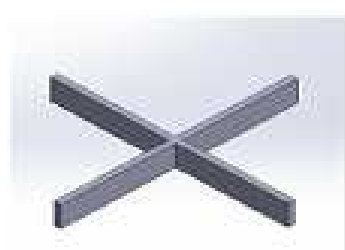

AVD-a[23]

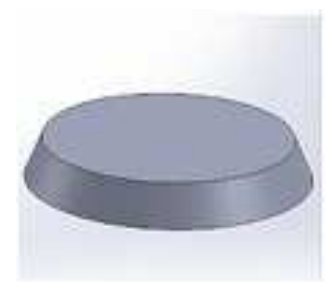

AVD-b[23]

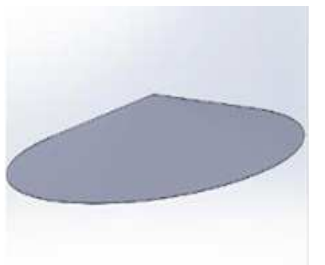

AVD-c[24]

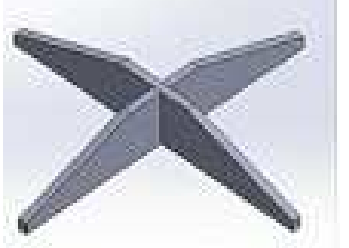

AVD-d[25]

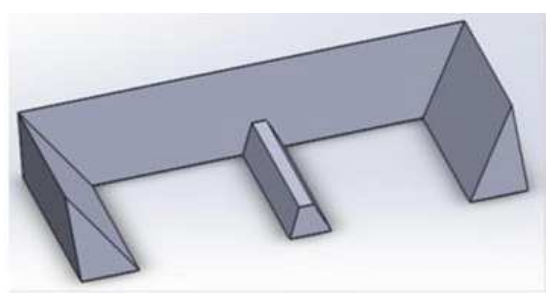

AVD-e [26]

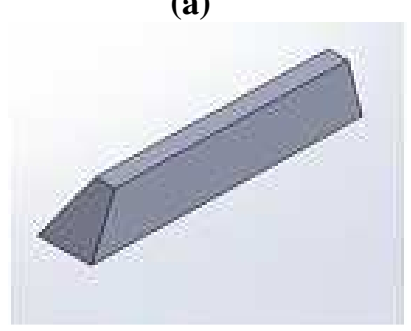

AVD-f [17]

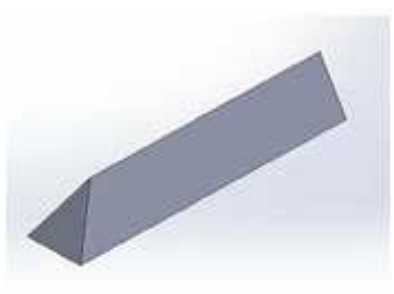

AVD-g[17]

(b)

Figure 3: Geometrical view of Anti Vortex Devices. (a) Axis Symmetry. (b) Floor Splitter. 


\subsection{Grid Selection}

We performed the comparative analysis for three types of grids on the numerical results based on [27]. Tetragonal mesh was used in case-I with total number of nodes as 1,60,423. In case-II more fine tetragonal mesh was used in case-II with total number of nodes as 5,40,097. In case-IV whole region was hexagonal with 8,80,754 nodes. Figure 4 shows the different arrangements of grids considered for comparison. During the transient simulation, top surface was visualized with fixed outlet velocity condition. At certain time, snapshots were taken to compare the movement at the top surface. Water surface was not smooth in case-I and case-II with the tetragonal mesh, which can be observed in Figure 5 (a)-(b). Moreover, velocity distribution was strongly disturbed with tetragonal mesh. And hence, disturbance in upstream section changed the size of surface vortex as compared to case-III in Figure 5(d). In our study, we will considered the hexagonal mesh for further study.

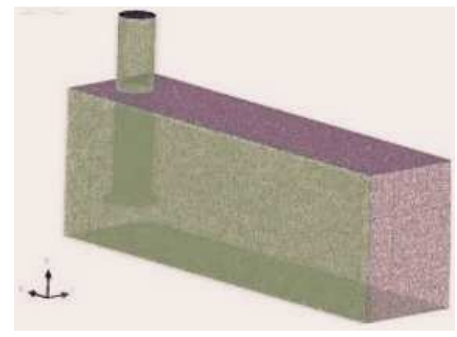

(a)

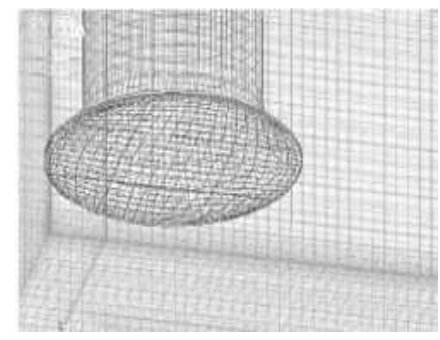

(b)

Figure 4: Mesh of Each Case. (a) Mesh Detail in Case-I;

(b) Mesh Detail at b:ell and su:mp in Case-IV

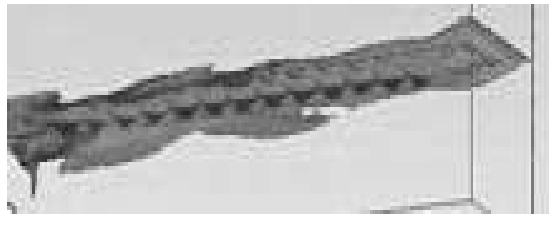

(a)

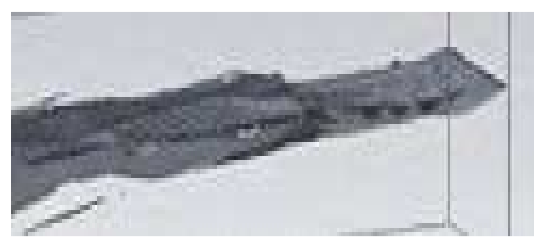

(b)

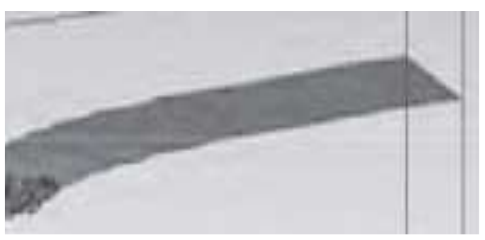

(c)

Figure 5: Visualization of Surface Movement in (a) Tetragonal Coarse Mesh

(b) Tetragonal Fine Mesh (c) Hexagonal Mesh with Fine Mesh.

\subsection{Governing Equation and Turbulence Model}

Navier-stokes equation can be solved with help of LES (Large eddy simulation) turbulence modeling. Large scale (grid scale) eddy motion and small scale turbulent fluctuation were solved by filtering out small scale eddy motion in LES. We can write the continuity and the momentum equation for selected model as,

$$
\begin{aligned}
& \frac{\partial \overline{u_{i}}}{\partial x_{i}}=0, \\
& \frac{\partial\left(\rho \overline{u_{i}}\right)}{\partial t}+\frac{\partial\left(\rho \overline{u_{i} u_{j}}\right)}{\partial x_{j}}=-\frac{\partial \bar{P}}{\partial x_{i}}+\frac{\partial\left(\overline{\tau_{i j}}\right)_{l}}{\partial x_{j}}+\frac{\partial\left(\overline{\tau_{i j}}\right)_{t}}{\partial x_{j}}+\rho g_{i},
\end{aligned}
$$

Where subscripts $\mathrm{i}, \mathrm{j}=1,2,3$ refer to the directions of $3 \mathrm{D}$ flows $\overline{u_{i}}=\mathrm{i}^{\text {th }}$ component of the filtered velocity field, $\bar{P}=$ filtered pressure, $\rho=$ density and $\mathrm{g}_{\mathrm{i}=} \mathrm{i}^{\text {th }}$ component of the gravitational field. $\left(\overline{\tau_{i j}}\right)_{l}=$ filtered molecular viscous 
stress tensor, $\left(\overline{\tau_{i j}}\right)_{t}=$ sub-grid scale Reynolds stress tensor field to be modeled. Smagorinsky model mostly used as subgrid scale model:

$$
\begin{aligned}
& \left(\overline{\tau_{i j}}\right)_{t}=\rho v_{t}\left(\frac{\partial \overline{u_{i}}}{\partial x_{j}}+\frac{\partial \overline{u_{j}}}{\partial x_{i}}\right) \\
& v_{t}=\text { sub-grid scale eddy viscosity. The near wall treatment is expressed as } \\
& \frac{v_{w}}{v_{t}}=k y_{w}^{+}\left(1-e^{\frac{-y_{w}^{+}}{A}}\right)^{2} \\
& y_{w}^{+}=\frac{y_{w} u_{\tau}}{v}
\end{aligned}
$$

Where $v_{w}=$ near wall eddy viscosity, $v=$ kinematic viscosity, $u_{\tau}=$ shear velocity, $\mathrm{k}=$ von Karman's constant ( $\mathrm{k}=0.41), \mathrm{A}=19$, and $y_{w}=$ distance from the solid wall to the center of the first cell adjacent to the wall.

\subsection{Boundary Condition}

A stable and uniform approach flow was considered as the inlet section far away from the inlet of the pipe. Only hydrostatic pressure distribution by the gravity force was considered in calculation domain. The Reynolds number $(\operatorname{Re})$ at reservoir inlet was $6.3 \times 10^{5}$. It was calculated as

$$
\operatorname{Re}=\left(\frac{\rho u D_{h}}{\mu}\right)
$$

where $D_{h}$, is the hydraulic diameter for the rectangular section. No slip condition was applied on the solid wall boundary. The outlet boundary conditions were set as constant mass flow rate, at the pipe outlet. A symmetry condition was taken for the open surface of reservoir. To completely resolve the complex shape of AVD and bell shaped intake, cut cell assembly meshing method was used to generate hexagonal mesh. In total $8 \times 10^{5}$ nodes were generated with inflation layer at solid wall. Fine mesh was used throughout the reservoir in order to accurately observe the formation of vortices and effect of AVD on vortex.

\subsection{Numerical Simulation}

A fully developed inlet flow with constant mass flow rate was assumed as mentioned by the Okamura et al [15] for the formation of steady surface vortex in their experimental results. The standard $k-\varepsilon$ turbulence model was applied to calculate steady state condition, then Large Eddy Simulation (LES) model was used to solve transient condition. LES provides more reliable results for such complex flows [28]. To validate the LES model, numerical results from the present study were compared with the experimental results of the Okamura et al [15] at line L085 passing through the centre of the intake section of pipe in the xy-plane at $\mathrm{z}=85 \mathrm{~mm}$ from the base of the sump as shown in Figure 6. 


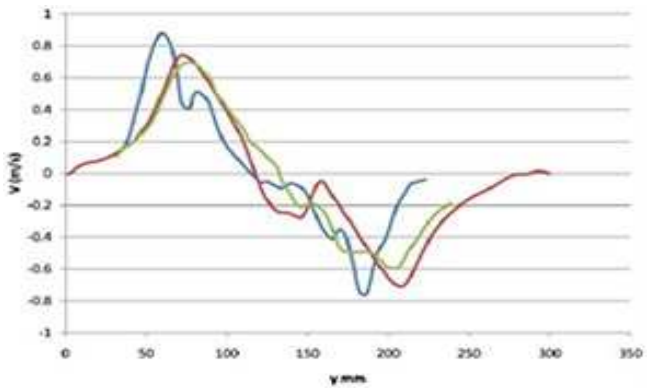

(a)

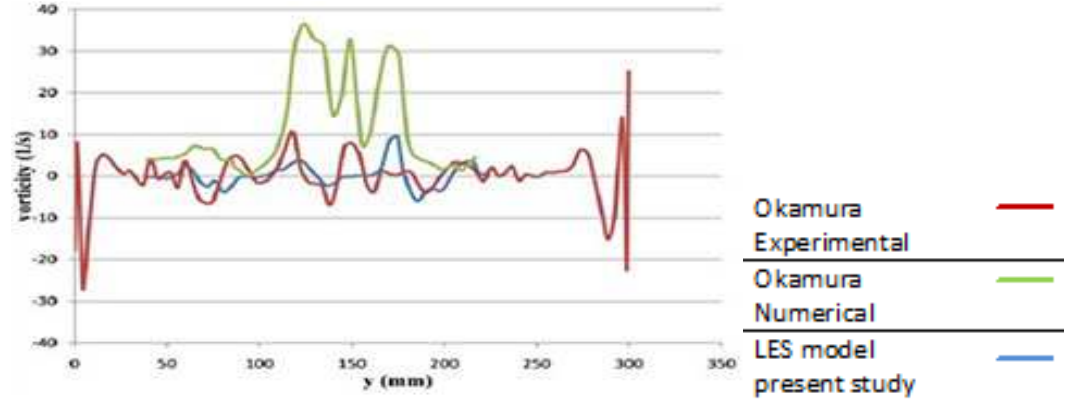

(c)

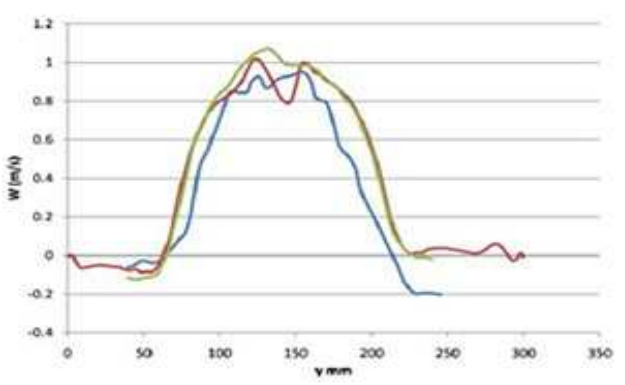

(b)

Figure 6: Comparison of LES Model Results in Present Study with Results from Okamura et al [15]. (a) y-Direction Velocity (b) z-Direction Velocity (c) Vorticity Value.

Velocity component in $\mathrm{y}$ and $\mathrm{z}$ direction are in great agreement with experimental results in comparison to the previous studies. Velocity component in x-direction is not taken into consideration as an error was reported by Okamural et al [15] in experimental results. Vorticity values for the present study is little higher at the center of the pipe but producing closer pattern in comparison to the previously used numerical model. Hence, we can say LES model used in this study can be used for reproducing the flow characteristics in the sump flow.

\section{RESULTS AND DISCUSSIONS}

Pump efficiency would be affected by a submerged vortex in the form of swirl flow and due to surface vortex which can lead to the entry of air parcel in the pump suction line. Flow conditions are selected in such a way where we can observe submerged and surface vortex. LES simulations are performed for assessment of the performance of all the AVD from (a) to (h) mentioned in the Figure 3. Same operating and numerical conditions are used in all the cases.

\subsection{Velocity Vector}

In the present case, both types of vortex can be observed in-spite of uniform flow condition at the inlet boundary conditions. Due to which, there is an increase in the vorticity at the intake section of pump. In order to reduce the vorticity different types of AVDs were tested. Velocity vector in terms of vorticity magnitude is shown in Figure 7 at plane Z095, $95 \mathrm{~mm}$ above the base of the sump. The plane Z095 is selected near the intake region of pipe to show the submerged and surface vortex. In Figure 7 (a) for the sump without AVD, both type of vortex can be observed with prominent surface vortex.

In Figure 7 (b)-(e) velocity vector for co-axial AVD-a to AVD-d are shown respectively. Reduction in submerged and surface vorticity can be seen with AVD-a and AVD-b. A displaced submerged vortex is observed with AVD-c. Large reduction in submerge vorticity can be noticed with AVD-d but there is not such reduction with surface vorticity. A clear presence of surface vortex can be observed at the periphery of the pipe. Velocity vector for floor splitter AVD-e to AVD-g 
is shown in Figure 7 (f) to (h) respectively. Increase in submerged vorticity is observed with AVD-e and surface vortex can also be seen in this case. Reduction in submerged and surface vorticity can be seen with AVD-f and AVD-g. A large reduction in surface vortex can be observed with AVD-g, but central submerged vortex divided in number of vortex in this case.

\subsection{Vorticity Magnitude}

Vorticity magnitude for all the cases plotted in Figure 8 at line L095 above $95 \mathrm{~mm}$ from the base and at line L660 which is at a distance 4D+Clearance from the base on xy-plane parallel to y-axis. Line L660 is located at a distance 4D from the entrance of the pipe. According to ANSI HI 1998/9.8 standards swirl angle is used to measure in the experiment at distance 4D from the entrance in the pipe. Angular velocity or spinning speed can be measured in the form of vorticity. Reduction in the value of the vorticity is observed at both the locations during the simulation for AVD-a, b, d, f and $g$ in comparison to the case without AVD. There is no significant reduction in the vorticity value at the entrance section of the pipe for AVD-c and AVD-e. But there is increase in the vorticity for AVD-c and AVD-e at line L660.

In Figure 8 at $\mathrm{x}$-axis point at $140 \mathrm{~mm}$ represent the central axis of the pipe. It can be observed the vorticity is higher at the locations away from the center. This may be due to the existence of high vorticity area between the side wall and the outer diameter. In case of less clearance, there may be a chance of high local velocities which can lead to higher vortex formation [29]. Flow separation at the wall of the pipe may be another possible reason for the high vorticity at this region. Due to this reason in most of the research work bell shaped pipe entrance is preferred over straight pipe. Based on the graph, it is confirmed that the installation of AVD either co-axial or floor splitter will reduce the vorticity value. For some shapes of AVD there can be a case of amplification of velocity component in the intake flow and hence form new vortices [30]. It can be observed in the case of AVD-c and AVD-e. In case of co-axial AVD a, b and d significant reduction in vorticity is observed with AVD-d. Co-axial types of AVD break the large vortex in the smaller values and hence reduced the vorticity. Similar in case of floor splitter AVD we observed significant reduction in AVD-g as compared to AVD-f as observed by Norizan et al [19]. Change in the cross section in terms of the reduction of the apex area from trapezoidal to triangular shape is the reason for this reduction.

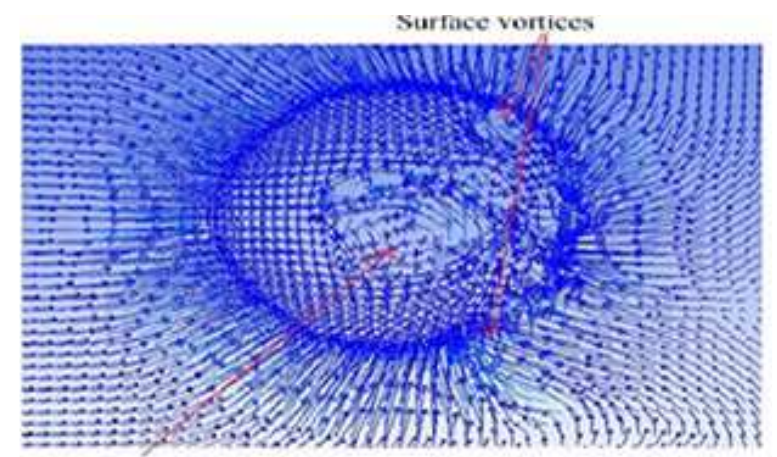

Submerged vortices

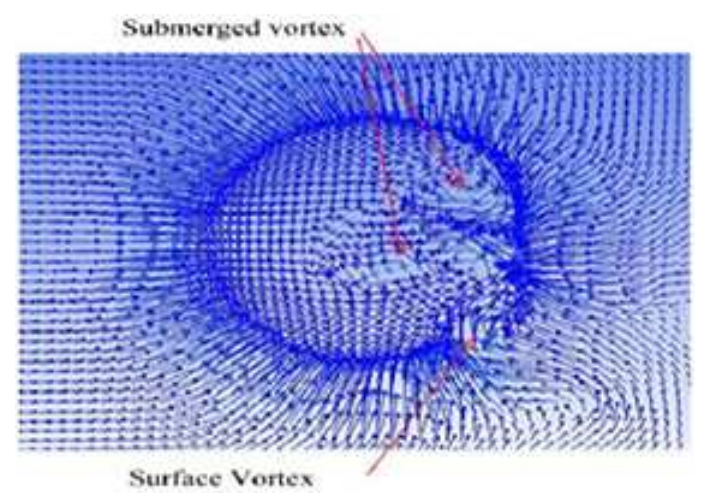

(b) 


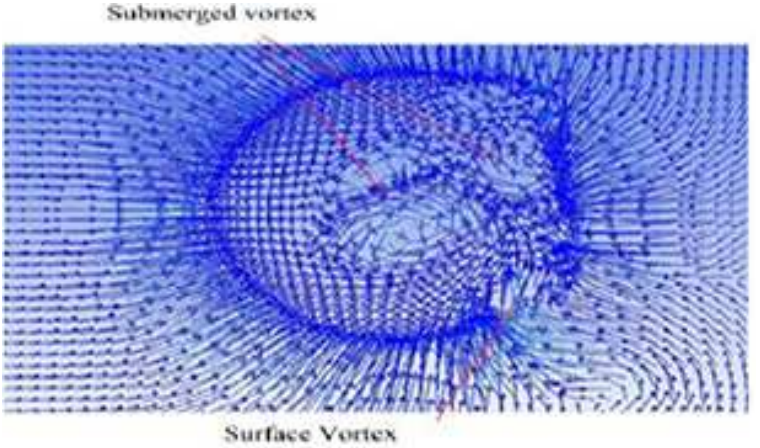

(c)

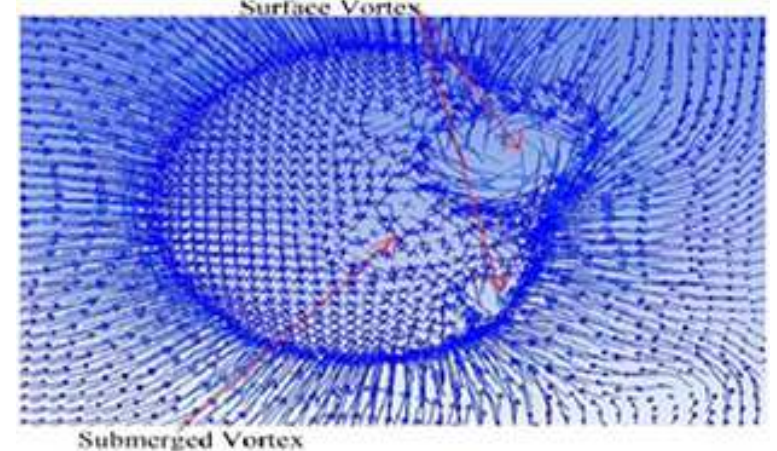

(e)

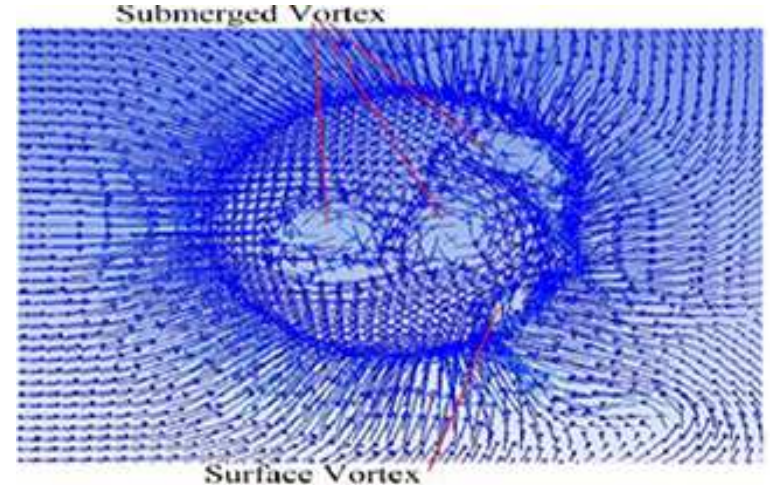

(g)

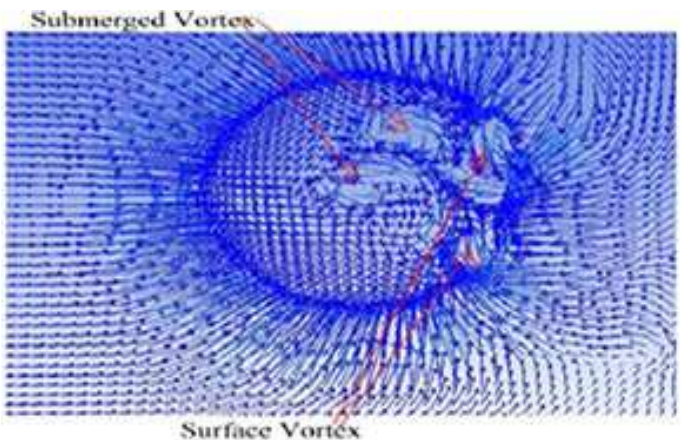

(d)

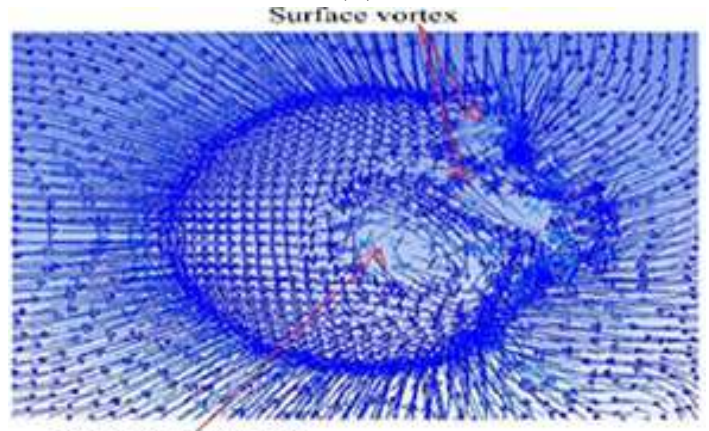

Submerged Vortex

(f)

Surface Vortex

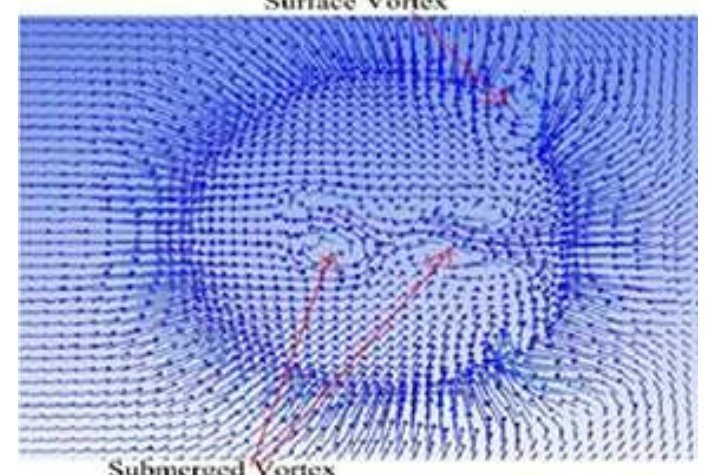

(h)

Figure 7: Velocity Vector in Terms of Vorticity Magnitude at Plane Z095.

(c) with AVD-b. (d) with AVD-c. (e)with AVD-d. (f)with AVD-e. (g)with AVD-f. (h) with AVD-g.

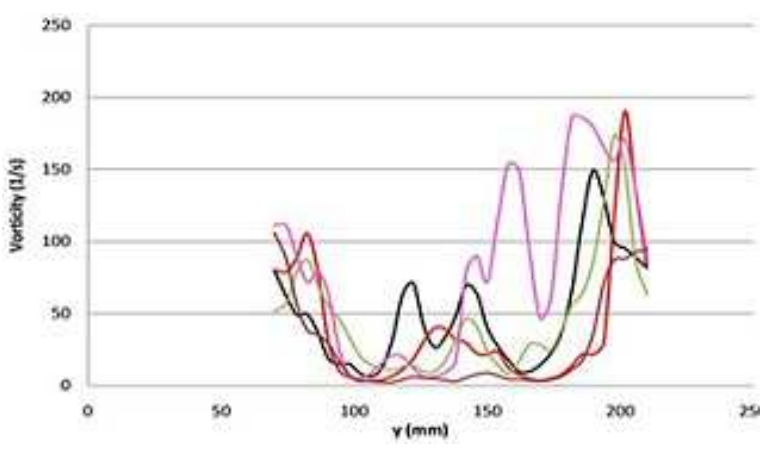

(a)

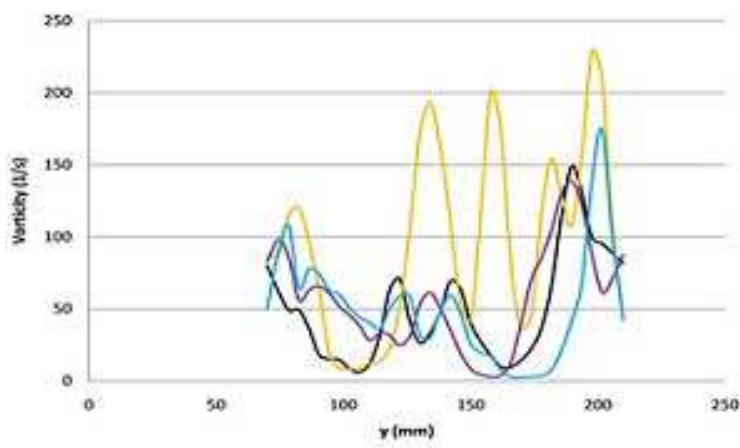

(b) 


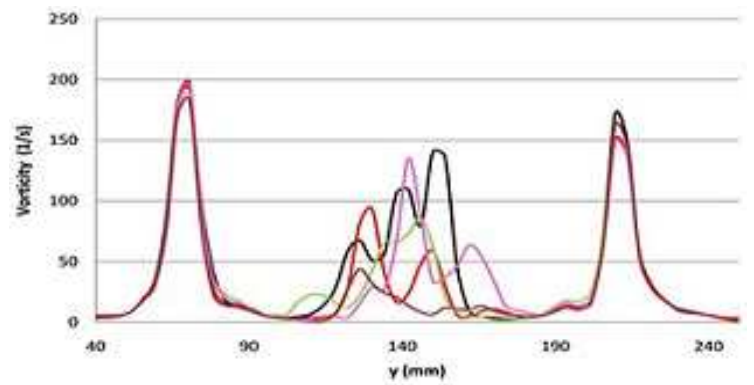

(c)

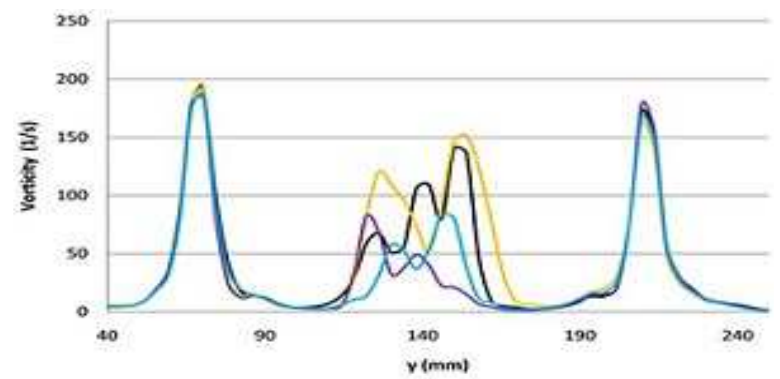

(d)

AVD-a ÂV-b,

AVD-c, AVD-d, AVD-e, Vorticity without AVD

AVD-f, AVD-G

Figure 8: Comparison of the Vorticity Magnitude with and without AVD.

(a) Vorticity for AVD-a, b, c and d at L660. (b) Vorticity for AVD-e, f and g at L660.

(c) Vorticity at AVD-a, b, c and d at L095. (d) Vorticity at AVD e, f and g at L095.

\subsection{Fast Fourier Transform Analysis}

For a complete assessment of the performance of the AVD, more clarification is required to explain the reduction capacity of the AVD. Based on the Figure 7, velocity vector diagrams and Figure 8 vorticity magnitude graphs AVD$\mathrm{d}$ and AVD-g are performed well in the present condition. As observed in Figure 7 (e) prominent surface vortex is observed in case of AVD-d, but it shows good reduction in the vorticity at intake section. For comparing the performance of all the AVD, pressure fluctuation is measured at the location of the surface vortex and FFT analysis is performed. The location of the point is $55.5 \mathrm{~mm}$ in $\mathrm{x}$-direction, $86 \mathrm{~mm}$ in y-direction and $229 \mathrm{~mm}$ in $\mathrm{z}$-direction at the centre of the vortices. MATLAB is used to do the FFT analysis of the data. Figure 9 shows the frequency spectra for the case without AVD and for all the AVDs. In all the frequency spectra Figure 9(h) shows the stable spectrum where there is single peak for AVD-g. Multiple peaks represent the more fluctuation during the flow time and hence show the tendency of the formation of the surface vortex. Figure 9(a) shows the frequency spectrum with predominant $5 \mathrm{~Hz}$, but it also has multiple peaks for high amplitude frequency. In the case of AVD-a Figure 9(b) a single predominant frequency of $7 \mathrm{~Hz}$ is observed. This means strength of surface vortex may be higher in this case but low number of peaks, as compared to case without AVD, so there will be fewer chances of surface vortices. For AVD-b, AVD-c and AVD-d predominant frequency of $4 \mathrm{~Hz}, 6 \mathrm{~Hz}$ and $3.5 \mathrm{~Hz}$ are observed in Figure 9 (c), (d) and (e) respectively. As shown in Figure 7(e) surface vortices is more prominent in the case of AVD-d, it can also be observed with the FFT analysis as multiple peaks are present in the frequency diagram with high amplitude; so there will be high chance for the formation of vortices in the sump. In case of floor splitter AVD Figure 9(f) shows multiple peaks with predominant frequency of $4 \mathrm{~Hz}$, hence high surface vortices will be observed as shown in Figure 7(f). In case of AVD-e and AVD-g we observed less fluctuation with predominant frequency of $3 \mathrm{~Hz}$ and $4 \mathrm{~Hz}$. Figure 9(h) indicates that the AVD-g reduce the surface vortex more prominently as less fluctuation is observed in comparison to other AVDs. 


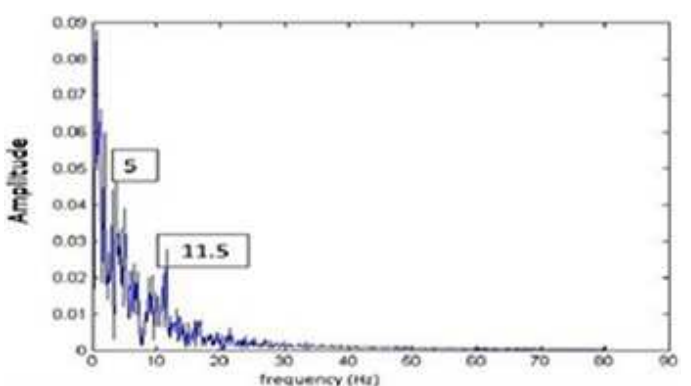

(a)

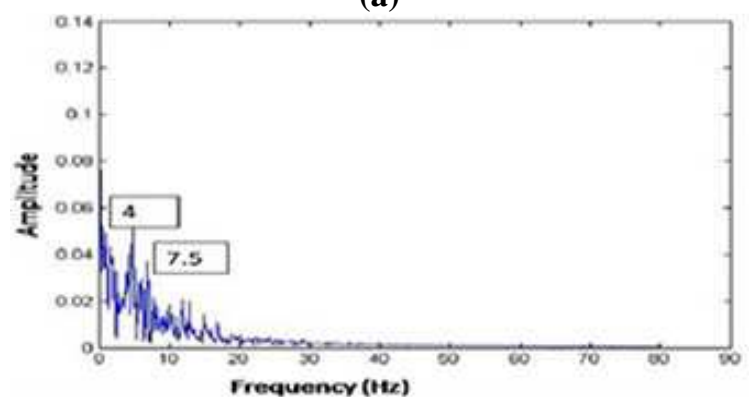

(c)

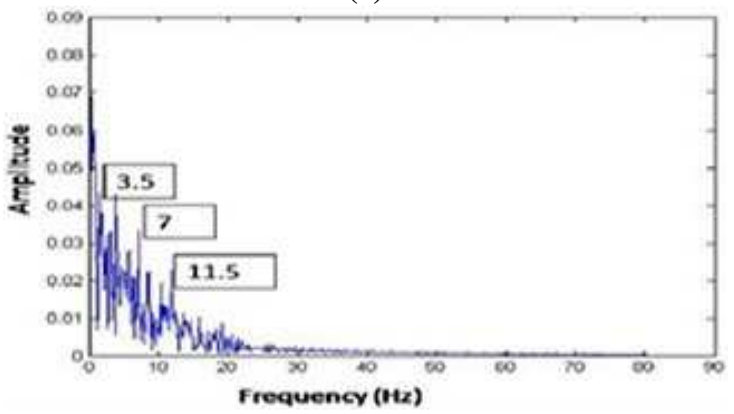

(e)

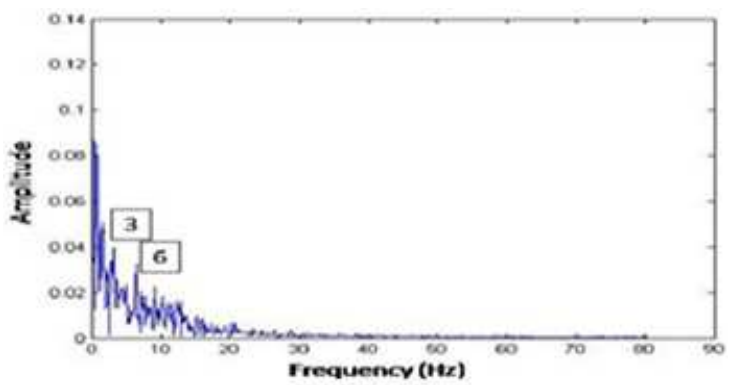

(g)

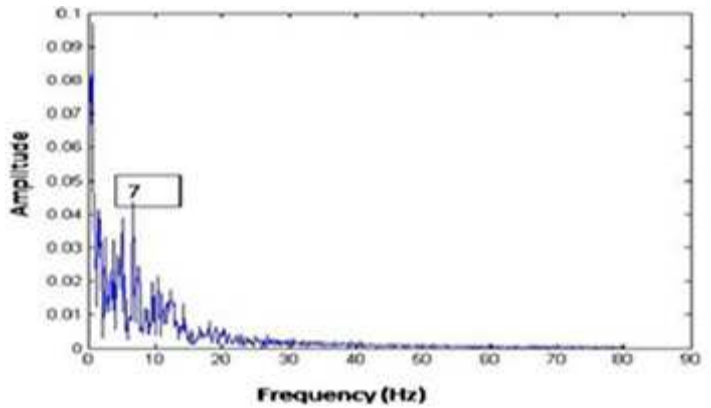

(b)

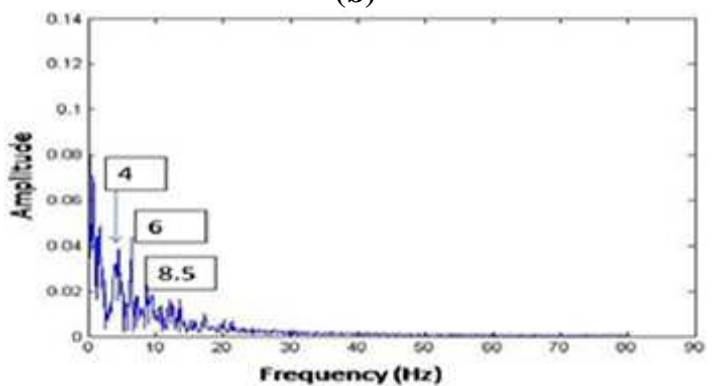

(d)

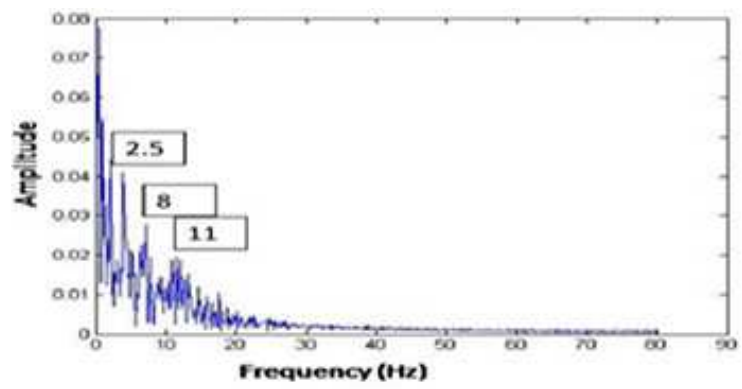

(f)

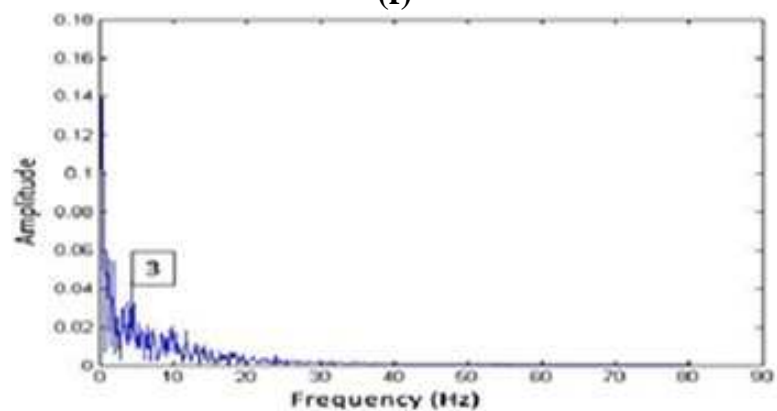

(h)

Figure 9: Frequency Spectra from FFT Analysis.(a) Without AVD.

(b) With AVD-a. (c) With AVD-b. (d) With AVD-c. (e) With AVD-d. (f) With AVD-e (g) With AVD-f (h) With AVD-g.

\section{CONCLUSIONS}

A numerical simulation was presented of flow in a pump sump with different types of Anti-Vortex Devices (AVD). Simulations were performed to compare the performance of different AVD in mitigation of air entrained vortex by Fast Fourier Transform (FFT) analysis. Four coaxial AVD and three floor splitter devices were used in the simulation. All the AVD were selected based on the performance of these devices in different operating conditions. In this study, a comparative analysis was performed to select the best AVD. The simulation was performed using large eddy simulation model with hexagonal mesh around 8 lakh nodes and it is validated by comparing the results with experimental data in the 
literature [15]. Pressure fluctuations were recorded during the transient simulation for 40 seconds at the location where surface vortex formed. Velocity vector and vorticity magnitude were compared for all the AVD against the condition with no AVD in the sump. Coaxial AVD were performed better in reducing the submerged vortex as compared to floor splitter devices which reduced the surface vortex. AVD of type-d displayed stronger reduction capacity towards submerged vortex and AVD type-g performed better in reducing the effect of surface vortex. To distinguish the performance of different AVD, FFT analysis found to be very useful. Higher value of characteristic frequency and large number of peaks indicated the large number of eddies in the sump. On the basis of FFT comparison, AVD type-g managed to reduce the surface vortex to large extent in the region around the intake.

These geometries can be reproduced while performing the experimental study for the same problem. New shape of AVD can also be developed and tested numerically with the help of FFT analysis. The results are beneficial in real life implementation in irrigation, drainage, and other discussed by [31].

\section{ACKNOWLEDGEMENT}

I would like to thank Dr C S Mathpati for valuable technical discussion and Dr Kuntal Chakrabarti for doing critical review. I would also like to thank Mr Amol Tare for helping in setting up the simulation lab.

\section{REFERENCES}

1. Bouzidi B. "New sizing method of PV water pumping station". Journal of Sustainable Energy Technologies and Assessments2013: 4: 1-10. DOI: 10.1016/j.seta.2013.08.004

2. Bartzas G, Komnitsas K. "Energy flow analysis in agriculture: the case of irrigatedpistachio production in Greece". Journal of Sustainable Energy Technologies and Assessments 2018: 28:73-80. DOI: 10.1016/j.seta.2018.06.007

3. European Commission, Study on improving the energy efficiency of pumps, technical Report, EuropeanCommission;2001.

4. Hydraulic Instutue, American National Standards For Pump Intake Design(ANSI/HI-9.8 1998); 1998.

5. British Standards Institution, Rotodynamic pumps Design of pump intakesRecommendations for installation of pumps (PD CEN/TR13930:2009);2009.

6. Japan Society of Mechanical Engineers, Standard method for model testing theperformance of a pump sump (JSME S004);1984.

7. Posser M. The hydraulic design of pump sump and turbine, British Hydromechanics Research Association/Construction Industry Research and Information Association; 1997.

8. Turbomachinery Society (TSJ) of Japan standardS2002:2005.

9. P. Kumar, R. P. Saini, "Study of cavitation in hydro turbines- A Review". Renewable and Sustainable Energy Reviews2010; 14; 374-383. DOI:10.1016/j.rser.2009.07.024

10. Rajasekaran, P., S. Vanangamudi, and P. Naveenchandran. "Automation of Hydraulic Tyre Wear Testing Machine using Proportional Control Valve by Interfacing Programming Logic Circuit."

11. Y. Zhang, K. Liu, H. Xian, X. Du. "A review of methods for vortex identification in hydro turbines". Renewable and Sustainable Energy Reviews2018;81;1269-85. https://doi.org/10.1016/j.rser.2017.05.058

12. S. Hwang Ahn, Y. Xiao, Z. Wang, X. Zhou, Y. Luo. "Numerical prediction on the effect of free surface vortex on intake flow characteristics for tidal power station”. Renewable Energy2017;101;617-28. https://doi.org/10.1016/j.renene.2016.09.021 
13. R. Goyal, B. K. Gandhi, M. J Cervantes, "PIV measurements in Francis Turbine- A Review and application to transient operations". Renewable and Sustainable Energy Reviews 2018;81; 2976-2991 DOI: 10.1016/j.rser.2017.06.108

14. Rajendran $n$ V. P., Contantinescu G. S., and Patel V. C. "Experiments on flow in amodel water-pump intake sump to validate a numerical model”. ASME, FEDSM98-5098, 1998.

15. Roh, H. W., Kim, J. S., and Suh, S. H. “Flow analysis within sump in a pump station using by the CFD.” Proceedings of the KSFM Conference 2002, pp.89-94.

16. Okamura T., Kamemoto K., Matsuji J. "CFD prediction and model experiment on suction vortices in pump sump". In: 9th Asian Conference on Fluid Machinery, Jeju, Korea,2007;1-10

17. Choi J, Choi Y, Kim C, Lee Y. "Flow uniformity in a multi intake pump sump model". Journal of Mechanical Science and Technologies 2010; 24 (7); 1389 - 1400. DOI 10.1007/s12206-010-0413-5

18. Kang W. Shin B. Doh D. "An effective shape of floor splitter for reducingsubsurface vortices in pump sump". Journal of Mechanical Science and Technologies2014; 28; 175-82. DOI:10.1007/s12206-013-1103-x

19. Yang F. Liu C. "Numerical and experimental investigations of vortex flows andvortex suppression schemes in the intake passage of pumping system". Advances in Mechanical Engineering 2014; 7(2); https://doi.org/10.1155/2014/547086

20. Viswam, Rahul, and S. Sankar. "Profile Modification of Wind Turbine Blade for Improving Efficiency."

21. Norizan T. A. Reda E. Harun z. "Enhancement of vorticity reduction by floor splitter inpump sump to improve pump efficiency". Sustainable Energy Technologies and Assessments 2018. 26; 28-36. https://doi.org/10.1016/j.seta.2017.06.001

22. Kim C. Choi Y. Lee Y. "A study on the effectiveness of an anti vortex device in thesump model by experiment and CFD". In: IOP Conference Series: Earth and Environment Science 2012. DOI:10.1088/1755-1315/15/7/072004

23. Padmanabhan M. and Hecker G. E. "Scale effect in pump sump models", Journal of theHydraulics Division $1984 ; 110$ (10);.1540-1556.

24. Jain A. K., RangaRaju K. G. and Garde R. J. "Vortex formation at vertical pipe intakes". Journal of the Hydraulic Division 1978; 104 (10); 1429 - 1445.

25. Tang X. L., Wang F. J., Li Y. J., Cong G. H., Shi X. Y., Wu Y. L., Qi L. Y. "Numerical Investigation of vortex flows and vortex suppression schemes in a large pumping-station sump”. Journal of Mechanical Engineering Science 2011; 225 (6);1459-80. DOI:10.1177/2041298310393447

26. Ramudu, V., U. Sudhakar, and P. Sadanandam. "Hydro Dynamic Performance of Francis Runner." power (MW) 500.800: 300 .

27. Yang F., Chao L. "Numerical and experimental investigations of vortex flows and vortex suppression schemes in the intake passage of pumping system". Hindawi publishing, Advances in Mechanical Engineering 2014; 7 (2); 547086. DOI: $10.1155 / 2014 / 547086$

28. Wang F. J., Zi D., Yao Z. F., Xiao R. F., Chen X., He C. L. "Numerical simulation on rectifying flow in intake system of a pumping station connected with headrace pipe". In: IOP Conference Series: Earth and Environment Science; 2016. 49(3); 032004. DOI:10.1088/1755-1315/49/3/032004

29. Kim H. J, Park S. W, Rhee D. S. "Numerical analysis of the effect of anti-vortex height onhydraulic performance of pump sump”. KSCE Journal of Civil Engineering2017; 21; 1484. https://doi.org/10.1007/s12205-016-0714-z.

30. Sugino Y., Kawakita K., Matsui J.," Numerical simulation on flow in pump sump with free surface”. 6th International 
Symposium on Fluid Machinery-2016(ISFMFE)

31. Mandapudi, Snigdha, et al. "CFD Simulation of Flow Past Wing Body Junction: A 3-D Approach." International Journal of Mechanical and Production Engineering Research and Development 7.4 (2017): 341-350.

32. Omori T, Jakirlic S, Tropea C. "Shearless and sheared flow past a circularcylinder: Comparative analysis by means of LES". International Journal of Heat and Fluid Flow2008; 29: 703-720. DOI: 10.1016/j.ijheatfluidflow.2008.03.012

33. Melville B, Ettema W, Nakato T. "Review of flow problems at water intake pumpsumps", Technical report, lowa institute of hydraulic research; 1994.

34. Tullis J. "Modeling in design of pumping pits". Journal of the Hydraulic Division 1979; 105(9);1053-63.

35. Marriappan P. Flood pumping station. IEM Bulletin 2016;15-9

\section{AUTHOR'S PROFILE}

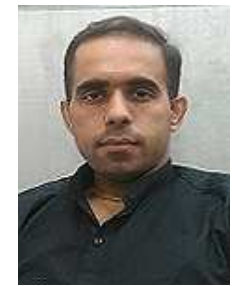

Rahul Paliwal received M.Chem degree from Institute of Chemical Technology in 2013. He worked as a Production Officer in Apcotex Industries from August 2010 to July 2011. He is currently working as an Assistant Professor and a Ph.D. candidate at the department of chemical engineering at NMIMS university, Mumbai. Mr Paliwal research interests are in the area of computational fluid dynamics, transport phenomenon and hydraulic machineries.

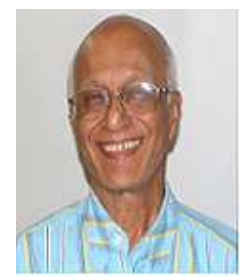

Anant S Jhaveri received Ph.D degree in chemical engineering from Mumbai university in 1968. He is currently associated with NMIMS university as visiting faculty after retiring from Head of Chemical Engineering Department. Mr Jhaveri research interests are in gas-liquid absorption, optimization of chemical operation, Non-Newtonian fluids, power consumption in fluid machinery. He has published 6 research papers. 
